\title{
Effects of Grain Size and Deformation Temperature on Hydrogen-Enhanced Vacancy Formation in Ni Alloys
}

Samantha K. Lawrence ${ }^{1 * \#}$, Yuriy Yagodzinskyy ${ }^{2}$, Hannu Hänninen ${ }^{2}$, Esa Korhonen ${ }^{3}$, Filip Tuomisto $^{3}$, Zachary D. Harris ${ }^{4}$, Brian P. Somerday ${ }^{5}$

${ }^{1}$ Sandia National Laboratories, Livermore CA, USA; ${ }^{2}$ Aalto University, School of Engineering, Espoo, Finland; ${ }^{3}$ Aalto University, School of Science, Espoo, Finland; ${ }^{4}$ University of Virginia, School of Engineering \& Applied Science, Charlottesville VA, USA, ${ }^{5}$ Southwest Research Institute, San Antonio, TX, "Present address: Los Alamos National Laboratory, Los Alamos, NM, \#Corresponding author: slawrence@lanl.gov

\begin{abstract}
Positron annihilation spectroscopy and thermal desorption spectroscopy experiments were combined to ascertain the role of hydrogen on generation of vacancies and vacancy clusters in Ni alloys. The effects of grain size and deformation temperature are emphasized for pure $\mathrm{Ni}$ single crystals and polycrystalline $\mathrm{Ni}-201$ alloy samples with two grain sizes that were thermally pre-charged with 3000 appm hydrogen. Variation in positron lifetime and intensity suggests that hydrogen enhances and stabilizes vacancies and vacancy clusters. Additionally, grain boundaries and the regions adjacent to them are preferential sites for vacancy and cluster formation. Hydrogen-altered vacancies and vacancy clusters are manifest in yield behavior differences: uniform vacancy distributions augment strength increases after hydrogen charging; enhanced yield strength during cryogenic deformation is ascribed to an 'Orowan type' strengthening mechanism while cross-slip restriction dominates hardening behavior at room temperature.
\end{abstract}

\section{Introduction}

Mitigating hydrogen-induced degradation of structural metals is a crucial step toward achieving safe and reliable delivery and storage of gaseous hydrogen as a transportation fuel. Hydrogen dissolved in metal causes degradation of mechanical properties, including reducing ductility and fracture toughness. A considerable number of research efforts have identified hydrogen-metal interactions that contribute to degradation, including the formation of hydrides [1], hydrogen adsorption-induced dislocation emission [2], plasticity effects [3], and alteration of cohesive energy [4]. Two degradation mechanisms are primarily invoked to frame hydrogen effects in metals where hydride formation is not favorable and the material contains internal 
hydrogen: hydrogen-enhanced localized plasticity (HELP) and hydrogen-enhanced decohesion (HEDE) [1]. The HELP theory suggests that hydrogen enhances the mobility of dislocations by shielding their interactions, thereby causing localized plasticity in regions of high hydrogen concentration, ultimately resulting in macroscopic brittle fracture via locally ductile processes $[3,5]$. In contrast, the HEDE mechanism assumes that dissolved hydrogen weakens interatomic bonds, and therefore the cohesive energy, resulting in brittle fracture modes (e.g. intergranular) [6-8]. Recently, a new mechanism has been proposed [9] that emphasizes the importance of vacancies in macroscale fracture processes. The hydrogen enhanced strain-induced vacancy (HESIV) mechanism suggests that hydrogen amplifies the concentration and agglomeration of vacancies by modifying their formation energy.

Vacancy concentrations far exceeding thermal equilibrium values have been detected for pure face centered cubic (FCC) metals, such as Pd [10] and Ni [11] subjected to high hydrogen pressure at temperatures ranging from $25^{\circ} \mathrm{C}$ to $800^{\circ} \mathrm{C}$. Similarly, plastic deformation stimulates non-equilibrium vacancy formation [12]. A variety of studies have assessed the synergistic effect of hydrogen and room temperature plastic deformation on vacancy generation and clustering. Positron annihilation spectroscopy (PAS) and thermal desorption spectroscopy (TDS) studies of deformed Fe [13], ferritic steels [14], and martensitic steels [15] reveal enhanced vacancy accumulation when the materials are hydrogen charged. Ultimately, magnified vacancy concentrations, and the clusters or voids formed by their agglomeration, are thought to promote crack propagation or amorphization [14], leading to hydrogen-related failure.

While a number of researchers have investigated temperature effects on yield behavior of $\mathrm{Ni}[16,17]$, these studies focused on the interaction of dislocations with solute hydrogen, namely the appearance and disappearance of the Portevin-Le Chatellier effect, rather than with hydrogen enhanced vacancies. Susceptibility of steels to HESIV-type failures depends not only on composition, but also on grain size [18]. The general effect of grain size remains unclear, however, because the grain refinement process alters boundary structure, which plays an important role in hydrogen-related failures. Only a few studies have investigated hydrogenenhanced strain-induced vacancy effects in FCC metals: Inconel 625 [19], Ni and Cu [20] and austenitic stainless steel [21]. Neither grain size nor deformation temperature effects on hydrogen-enhanced vacancy generation and coalescence have been studied in detail for FCC metals. 
Advancing the current understanding of synergy between hydrogen- and strain-induced vacancy formation in FCC metals requires decoupling the interplay among grain size, deformation temperature, and hydrogen charging condition in commercially pure alloys. Positron annihilation spectroscopy is an efficient tool for interrogating the effect of hydrogen and plastic deformation on vacancies and their complexes because positrons are trapped at lattice defects, such as vacancies and vacancy clusters [22]. Positron annihilation is quantified by the positron lifetime, which correlates with the magnitude of the electron density at the site of positron annihilation and gives information about the open volumes at vacancies. Positron lifetime in a monovacancy is about $50 \%$ longer than in the perfect bulk and increases further when vacancies cluster into three-dimensional agglomerates [23]. Experimental and computational investigations reveal marked variation in positron lifetime after hydrogen charging [24,25] or plastic deformation [26,27] of $\mathrm{Ni}$, suggesting PAS is an ideal method for interrogating the combined effect of hydrogen charging and plastic deformation on defect structure evolution in $\mathrm{Ni}$, as a function of grain size and deformation temperature.

The objective of the current study is to ascertain the role of grain size and deformation temperature on generation of vacancies and their complexes in hydrogen-charged and deformed Ni alloys. To achieve this goal, we have combined PAS of as-supplied, hydrogen-charged, asdeformed, and charged + deformed specimens with TDS, as an indicator of defect structure evolution. Commercially pure Ni-201 specimens with two grain sizes (nominally $35 \mu \mathrm{m}$ and 1 $\mathrm{mm}$ ) were mechanically deformed at two temperatures ( $293 \mathrm{~K}$ and $77 \mathrm{~K}$ ) to examine the effect of grain size and deformation temperature on defect generation with and without hydrogen precharging. Single crystals of $99.999 \%$ pure Ni were used in the study as reference material.

\section{Experimental Procedures}

\subsection{Material Preparation}

A commercially pure nickel alloy, Ni-201, was used for this investigation. Thyssen Krupp manufactured two plates with different grain sizes. Plate 1 (thickness $=127 \mathrm{~mm}$ ) had an as-supplied grain size of $35 \mu \mathrm{m}$ and impurities (in wt.\%) of $0.01 \mathrm{C}, 0.09 \mathrm{Mn}, 0.062 \mathrm{Fe}, 0.046 \mathrm{Si}$, $0.026 \mathrm{Co}, 0.022 \mathrm{Mg}, 0.021 \mathrm{Al},<0.01 \mathrm{Cu}$, and $<0.001 \mathrm{~S}$. Plate $2($ thickness $=254 \mathrm{~mm})$ had an as-supplied grain size of $200 \mu \mathrm{m}$ and impurities (in wt.\%) of $0.01 \mathrm{C}, 0.01 \mathrm{Mn},<0.01 \mathrm{Fe}, 0.05 \mathrm{Si}$, 
$<0.01 \mathrm{Cu}$, and $<0.001 \mathrm{~S}$. Grain boundary segregation of impurities, especially of $\mathrm{S}$ in $\mathrm{Ni}$, has been shown to alter the amount of hydrogen required to achieve 100\% intergranular fracture and can impact localized plasticity prior to failure [28-30]; to mitigate this issue, an alloy grade with an ultralow sulfur concentration was chosen for this investigation. The as-supplied Ni-201 Plate 2 was annealed at $900^{\circ} \mathrm{C}$ for $1 \mathrm{~h}$, stimulating grain growth; this heat treatment resulted in a grain size of $1 \mathrm{~mm}$. Three sets of specimens were extracted from each plate: PAS specimens (dimensions: $4 \mathrm{~mm}$ x $4 \mathrm{~mm}$ x $0.5 \mathrm{~mm}$ ), TDS specimens (dimensions: $4 \mathrm{~mm}$ x $6 \mathrm{~mm} \times 0.3 \mathrm{~mm}$ ) and tensile specimens (gauge diameter $4 \mathrm{~mm}$, gauge length $12.7 \mathrm{~mm}$ ). After tensile straining, specimens for PAS and TDS were extracted from the gauge section of the tensile bars such that the thickness dimension was parallel to the tensile axis. Surfaces were finished with emery paper No. 2400.

Pure, $99.999 \%$ nickel single crystals (SCs) were supplied by MaTecK GMBH (Germany) and were use as reference material in order to elucidate the specific impact of grain boundaries on TDS and PAS data of nickel with and without hydrogen as well as their contribution to mechanical behavior of hydrogen-charged nickel. The TDS and PAS specimens (dimensions: 3.5 $\mathrm{mm} \times 4 \mathrm{~mm} \times 0.75 \mathrm{~mm}$ ) were cut from SC plate with the (011) plane parallel to the largest side. Sub-sized tensile test specimens (gauge dimensions: $0.75 \mathrm{~mm}$ x $3.5 \mathrm{~mm}$ x $10 \mathrm{~mm}$ ) with loading axis along [001] crystallographic direction were cut from the same SC plates. TDS and PAS specimens were cut from the gauge length after straining to about $10 \%$ elongation.

\subsection{Thermal Desorption Spectroscopy}

Hydrogen thermal desorption spectra were obtained at a constant heating rate of $6 \mathrm{~K}$ $\min ^{-1}$ in the temperature range from 298 to $1123 \mathrm{~K}$ by measuring the hydrogen partial pressure in the UHV chamber of a TDS apparatus. The basic vacuum in the apparatus was kept better than $10^{-4} \mathrm{~Pa}$ [31]. After calibration of the TDS apparatus the partial pressure data were re-calculated to hydrogen desorption rates in atomic $\mathrm{ppm} \mathrm{s}^{-1}$ vs. temperature of the linear heating [31].

\subsection{Positron Annihilation Spectroscopy}

Positron annihilation spectroscopy (PAS) measurements were carried out with the positron lifetime method using a conventional fast-fast spectrometer in collinear geometry $[23,24]$ with a time resolution of $250 \mathrm{ps}$. In this scheme, the positron source, $\mathrm{a}^{22} \mathrm{Na}$ salt 
deposited on 1.5- $\mu \mathrm{m}$ thick metal foil, is sandwiched between two identical Ni specimen pieces. The lifetime spectrum was analyzed as the sum of the exponential decay components convoluted with the Gaussian resolution function of the spectrometer after subtracting the constant background and annihilations in the source material.

Note that while other researchers [32] have discussed positron trapping at grain boundaries, it is unlikely in this case. The effective diffusion distance for positrons in a perfect lattice is about $0.25 \mu \mathrm{m}$, so the relative probing volume of grain boundaries by positrons would be less than $1 \%$ for $35 \mu \mathrm{m}$ grains, assuming a perfect lattice. However, because the Ni-201 grains under consideration here are far from perfect, the probing volume would be smaller. Thus, the likelihood that positrons are efficiently trapped by grain boundaries that have not been modified by hydrogen charging or plastic deformation is substantially reduced. The excess free volume generated when grain boundary structure is modified by vacancy formation and coalescence, however, readily traps positrons.

\subsection{Tensile Straining}

Uniaxial tensile tests were conducted on as-supplied/as-annealed and hydrogen pre-charged $\mathrm{Ni}-201$ specimens both at room temperature $(293 \mathrm{~K})$ and in a liquid nitrogen bath $(77 \mathrm{~K})$. To ensure equilibrium grain boundary hydrogen concentrations, all the tensile bars were allowed to age at room temperature for one hour prior to testing. Tensile deformation was performed using an MTS 810 servo-hydraulic mechanical testing system under actuator displacement control at a rate of $0.01 \mathrm{~mm} / \mathrm{s}$ (initial engineering strain rate $=6.3 \times 10^{-4} \mathrm{~s}^{-1}$ ). Displacement was controlled such that the specimens were strained to about $10 \%$. Elongation was uniform and plastic strain was verified by calculating axial strain based on elongation as well as reduction in area after straining, $R A=\left(d_{i}^{2}-d_{f}^{2}\right) / d_{i}^{2}$, where $d_{i}$ is the initial diameter of the gauge section, and $d_{f}$ is the final diameter after tensile straining. Total strains ranged between $8 \%$ and $11 \%$ and are consistent between extensometer measurements and $R A$ calculations.

Tensile testing of as-supplied and hydrogen-charged SC specimens was performed with a 35 kN MTS Insight electromechanical testing system at $293 \mathrm{~K}$ only. Using a strain rate of $5 \times 10^{-4} \mathrm{~s}^{-}$

${ }^{1}$ samples were deformed to 6.5 and $10 \%$ elongation. 


\subsection{Hydrogen Charging}

Hydrogen was dissolved into the pre-machined PAS and TDS specimens and the tensile bars by thermally charging in high-pressure hydrogen gas. The specimens were inserted into a thick-walled A-286 stainless steel pressure vessel and then placed in a furnace. Residual gases were removed from the sealed pressure vessel and gas-handling manifold using a purging and evacuating sequence. Three purge/evacuation cycles were conducted with helium, followed by three cycles with hydrogen. Once the purging/evacuation cycles were completed, the pressure vessel was heated and filled with $99.9999 \%$ hydrogen gas. Charging was conducted at elevated temperature to enhance hydrogen diffusion, thereby minimizing charging times, but temperatures were limited to avoid thermally activated microstructural changes in the nickel. Specimens were charged at a hydrogen gas pressure of $62 \mathrm{MPa}$ and a temperature of $573 \mathrm{~K}\left(300^{\circ} \mathrm{C}\right)$ for $144 \mathrm{hrs}$ to achieve a uniform hydrogen concentration of about 3000 atomic ppm (appm). Hydrogen-charged specimens were stored at $223 \mathrm{~K}\left(-50^{\circ} \mathrm{C}\right)$ and transported cryogenically to minimize hydrogen loss.

\subsection{Testing Procedure}

PAS and TDS measurements were carried out for samples machined directly from the assupplied or as-annealed Ni-201 plates and from as-supplied SC plate; PAS was conducted using a $\mathrm{Cu}$ foil source. The specimens, as well as the tensile bars were then thermally pre-charged with hydrogen following the procedure outlined above. Upon removal from the pressure vessel the specimens were immediately stored in a cryogenic freezer. After charging, the Ni-201 tensile specimens were strained to $10 \%$ either at room temperature or $77 \mathrm{~K}$. PAS and TDS specimens were extracted from the gauge length of the tensile specimens. Disks with a thickness of 0.75 $\mathrm{mm}$ were cut in the direction transverse to the loading axis from the Ni-201 specimens and directly from the gauge of SC tensile specimens and then polished to achieve a final thickness of $0.5 \mathrm{~mm}$ for PAS and TDS. Specimens were stored cryogenically between all preparation steps.

In addition to hydrogen-charged tensile specimens, as-supplied/as-annealed tensile specimens were strained to $10 \%$ at either RT or $77 \mathrm{~K}$, and then PAS and TDS specimens were extracted from the gauge section of the as-strained tensile bars as described above. PAS and TDS measurements were subsequently conducted for all the hydrogen-charged and non-charged specimens. 


\section{Results}

\subsection{Thermal Desorption Spectroscopy}

Thermal desorption rates of hydrogen as a function of desorption temperature for undeformed SC Ni specimens and pre-machined polycrystalline Ni-201 TDS coupons are shown in Figure 1a while spectra for the hydrogen-charged and strained Ni SC and Ni-201 specimens are shown in Figure 1b. The TDS indicates that the total dissolved hydrogen content in the undeformed SC Ni specimens and pre-machined Ni-201 TDS coupon specimens is on the order of 300 to $500 \mathrm{appm}$. This value is less than the target hydrogen content of $3000 \mathrm{appm}$. However, integrating the data in Figure 1b indicates that the hydrogen concentration in coupons extracted from the strained $\mathrm{Ni}-201$ tensile samples is on the order of $3000 \mathrm{appm}$. Conversely, integrating the data for the sub-sized SC tensile samples in Figure $1 \mathrm{~b}$ results in a lower hydrogen concentration. The hydrogen charging conditions likely attained the target concentration of 3000 appm during charging and cryogenic specimen storage was effective in retaining the dissolved hydrogen after removal from the charging vessel. However, rapid hydrogen egress at high temperatures from the thin plate TDS specimens and Ni SC tensile specimens during the 16-hour cooling period of the pressure vessel after charging allows some amount of hydrogen outgassing.

A prominent desorption peak at about $600 \mathrm{~K}$ is observed for all hydrogen-charged specimens. In addition, a secondary desorption peak, at about $750 \mathrm{~K}$, develops for the precharged 35- $\mu \mathrm{m}$ grain size Ni-201 and 1-mm grain size Ni-201 alloys, but the peak does not appear in the SC specimen implicating hydrogen release from grain boundary trapping sites at $750 \mathrm{~K}$ (Figure 1a). This secondary peak shifts to about $770 \mathrm{~K}$ for samples that were hydrogen charged and subsequently strained (Figure 1b). The peak is particularly pronounced for the 1-mm grain size sample strained at $77 \mathrm{~K}$. The location and amplitude of this second peak generally corresponds to hydrogen release from stronger trap sites, such as vacancy clusters, induced by deformation in the presence of hydrogen. The secondary peak is not observed for the SC specimen strained at $293 \mathrm{~K}$, hence it is assumed that straining modifies the trapping sites located on and/or caused by grain boundaries. The minor TDS peak was also missing in the hydrogencharged 1-mm grain size $\mathrm{Ni}-201$ specimen strained at $293 \mathrm{~K}$, but its lack in this case is still unclear. Detailed analyses of the minor TDS peak origin and its fine structure need further efforts. 


\subsection{Positron Annihilation Spectroscopy}

Positron annihilation spectra are characterized by both positron lifetime and lifetime intensity. Positron lifetime, $\tau$, correlates with the magnitude of the electron density at the annihilation site and gives information about the open volume at defects while lifetime intensity, $I$, is an indication of defect concentration. Figure 2 shows the positron lifetime spectra measured in $5 \mathrm{~N} \mathrm{Ni}$ single crystals in the as-supplied state and after deformation at $293 \mathrm{~K}$ with and without hydrogen pre-charging. All the spectra are decomposed into two components, with the intensity of the longer (second) component increasing with the amount of processing. In addition, the longer (second) component is longer in the sample that is both pre-charged with hydrogen and deformed. Similar spectra were obtained for Ni-201 alloy samples. Fitting of these spectra provides positron lifetime values. The results of the PAS measurements as a function of processing conditions for the different $\mathrm{Ni}$ samples are presented in terms of the average positron lifetime values, $\tau_{\text {ave }}$ (Figure $3 \mathrm{a}$ ), the short and long positron lifetime components, $\tau_{1}$ and $\tau_{2}$ (Figure 3b, 3c, Table I), and the long-term lifetime component intensity $I_{2}$ (Table I).

Average positron lifetime response as a function of measurement time is plotted for both non-charged and hydrogen-charged specimens deformed at $77 \mathrm{~K}$ in Figure 3a. Lifetime values (collected for all samples in Table I) for hydrogen-charged specimens correspond more closely with that for vacancies in $\mathrm{Ni}$ (180 ps for monovacancies, 210-340 ps for vacancy clusters [33]) compared to dislocations (160 ps) [33]. Additionally, in the case of the SC and the unstrained Ni201 samples, it is unlikely that new dislocations have been generated in the lattice as a result of hydrogen charging. Therefore, the defects most probably responsible for trapping positrons are vacancies and vacancy clusters.

The positron lifetime spectrum for all the measured samples can be separated into two components, a short lifetime component, $\tau_{1}$, and a long lifetime component, $\tau_{2}$ (Table I). The $\mathrm{SC}$ sample in the as-supplied state manifests a short lifetime component of $116 \mathrm{ps}$, which corresponds well with literature data for positron lifetime in bulk pure Ni [34]. The short lifetime component in SC samples increases to values between $125 \mathrm{ps}$ and $132 \mathrm{ps}$ in the presence of hydrogen. The $\tau_{1}$ value in deformed SC samples remains the same as in the hydrogen-charged condition (about $125 \mathrm{ps}$ ), but the corresponding intensity is considerably lower compared to the hydrogen-charged specimen. 
Considering the coarse-grained and fine-grained Ni-201 alloys, the as-supplied lifetime values are generally longer than those of the pure Ni SC, suggesting impurities in the commercially pure composition may increase the measured positron lifetime. The $\tau_{1}$ values for as-supplied/as-annealed coarse-grained and fine-grained Ni-201 alloys are both about 120 ps (see Table I). Plastic deformation shifts the short lifetime component of both coarse- and fine-grained Ni-201 to the range of 160 to 167 ps. Straining of the Ni-201 alloy samples pre-charged with hydrogen results in a $\tau_{1}$ value close to $166 \mathrm{ps}$ for both deformation temperatures. Hydrogen trapped in a vacancy is known to significantly reduce the apparent positron lifetime in a $\mathrm{Ni}$ monovacancy [34,35] (the typical H-free value is about $180 \mathrm{ps}$ ); in this case, then, the observed $\tau_{1}$ value of $166 \mathrm{ps}$ after straining in the presence of hydrogen most likely corresponds to positron annihilation at monovacancies containing hydrogen atoms.

The long lifetime component, $\tau_{2}$, also varies with material and processing condition. A $\tau_{2}$ value of $240 \mathrm{ps}$, with a small intensity, was measured in the as-supplied Ni SC. Though this value falls in the range of vacancy clusters (which might have formed in the Ni lattice during SC growth) the small intensity implies very few of these defects are initially present in the microstructure. Hydrogen charging of the $\mathrm{Ni} \mathrm{SC}$ results in a pronounced decrease in $\tau_{2}$, but an increased intensity, as compared with the as-supplied state. Similarly, straining of non-charged SC samples at $293 \mathrm{~K}$ also reduces the $\tau_{2}$ value, but the intensity values are nearly triple those measured in the as-supplied samples. Increasing intensities are linked with hydrogen and deformation-enhanced increases in the vacancy cluster concentration.

The long lifetime values for hydrogen-charged Ni-201 specimens range from 215 ps to 255 ps and those for samples strained without hydrogen range from 247 ps to 303 ps (see Table I). These values are longer than those measured in the as-suppled/as-annealed conditions, revealing that hydrogen charging and plastic deformation independently enhance vacancy clustering in the Ni SC and both Ni-201 alloys.

Straining of hydrogen pre-charged samples results in a substantial increase of the longterm lifetime component in all cases; the value of $\tau_{2}$ is close to $310 \mathrm{ps}$ in both Ni-201 alloys, while it is about 280 ps in the strained Ni SC sample (see Table I). Additionally, the increase in $\tau_{2}$ from the hydrogen-charged condition to the hydrogen-charged + strained condition is considerably larger for the $35-\mu \mathrm{m}$ grain size alloy than for either the 1-mm grain size or SC samples $\left(\Delta \tau_{35 \mu m}=98, \Delta \tau_{1 m m}=52, \Delta \tau_{S C}=40\right)$. This finding indicates that grain boundaries in 
Ni play a specific role in free volume generation during plastic deformation in the presence of hydrogen. Lastly, testing temperature does not affect the $\tau_{2}$ values in hydrogen-charged and strained Ni-201 alloys; however, the corresponding relative intensity, $I_{2}$, appears somewhat higher for low-temperature tests (see Table I).

\subsection{Tensile Straining}

Displacement-controlled tensile straining up to about $10 \%$ resulted in uniform deformation in the gauge section of the Ni-201 alloy tensile specimens. A $0.2 \%$ offset criterion was applied to calculate yield strength from the resulting stress-strain curves, which are presented for samples deformed at $293 \mathrm{~K}$ and $77 \mathrm{~K}$ in Figure $4 \mathrm{a}$ and Figure 4b, respectively. In general, yield strength is lower for the 1-mm grain size material than the $35-\mu \mathrm{m}$ grain size material, as may be expected by the Hall-Petch relationship and the well-annealed condition of the 1-mm grain size material. For both the $1-\mathrm{mm}$ grain size and $35-\mu \mathrm{m}$ grain size materials, hydrogen charging leads to an increase in yield strength and an increased work hardening rate, calculated as the slope of the stress-strain curve at true strains of $6 \%$ and $8 \%$. The yield strength change is most pronounced for specimens strained at $77 \mathrm{~K}$ (Fig. 4b), with an approximately $18 \%$ increase for the 1-mm grain size material and a approximately $50 \%$ increase for the 35 - $\mu \mathrm{m}$ grain size material (see Table II). The work hardening increases with hydrogen charging and it is most pronounced during deformation at room temperature (see Fig. 4a and Table II),

Ni SC tensile samples with loading axes close to the [001] crystallographic direction were strained to about $7 \%$ in tension at $293 \mathrm{~K}$ only; corresponding stress-strain curves are shown in Figure 4a. Hydrogen charging leads to significant increases in both the yield strength (42\%) and work hardening rates (approximately 50\%).

\section{Discussion}

Several key trends in PAS data are observed with respect to positron lifetime, yielding information about the type and size of the positron trapping sites, and the relative lifetime component intensity, indicating the trapping site concentration. Recall that the positron lifetime

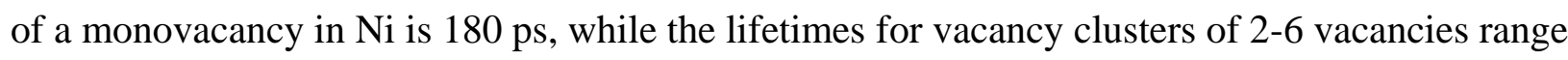
between 210 and 340 ps, increasing with the cluster size [33]. However, when vacancies or their 
clusters contain trapped hydrogen the positron lifetime decreases substantially due to a decrease in the apparent free volume.

For this analysis, the average positron lifetime was decomposed into two components, since $\tau_{\text {ave }}=\sum_{i} \tau_{i} I_{i}$. The short lifetime, $\tau_{1}$ corresponds to positron annihilation in the crystal lattice bulk, and/or at monovacancies, and/or dislocation cores [27], while the long lifetime, $\tau_{2}$, corresponds to annihilation at larger vacancy clusters [20]. Notably, $\tau_{\text {ave }}$ values of hydrogencharged 1-mm grain size and 35- $\mu \mathrm{m}$ grain size $\mathrm{Ni}-201$ alloy specimens evolve with time (see Figure 3a). The observed evolution occurs after deformation at $77 \mathrm{~K}$ and at $293 \mathrm{~K}$, but the effect is more pronounced after low-temperature straining, hence only these data are shown in Figure 3a. The values of $\tau_{\text {ave }}$ increase with measurement duration reflecting a few possible processes: hydrogen release from the trapping sites and desorption from the specimen due to relatively fast diffusion, an increase of the positron trapping site free volume due to vacancy agglomeration, and/or an increase of the trapping site density. In contrast, the hydrogen-charged SCs deformed at $293 \mathrm{~K}$ do not display time dependence, suggesting that grain boundaries play a key role in the observed evolution of $\tau_{\text {ave }}$, and therefore, hydrogen-enhanced vacancy processes.

The increase in $\tau_{1}$ and $I_{1}$ values of hydrogen-charged Ni-201 alloys, compared with the as-supplied/as-annealed states indicates that hydrogen enhances monovacancy formation and subsequently stabilizes the vacancies. Additionally, an increase in $\tau_{2}$ with a corresponding decrease in $I_{2}$ suggests that hydrogen enhances vacancy clustering, resulting in larger clusters, but a decreased cluster quantity. Ab-initio calculations of hydrogen-vacancy interactions in $\mathrm{Ni}$, performed by Tanguy et al. [36] support the assessment that thermally activated vacancies in the presence of hydrogen have a tendency to agglomerate into vacancy complexes. The authors found that the maximum binding energy for a monovacancy-hydrogen pair $\left(\mathrm{VH}_{1}\right)$ is $0.27 \mathrm{eV}$; this value increased to $0.41 \mathrm{eV}$ for a di-vacancy-hydrogen complex. TDS data further support the PAS results; a small peak develops around $750 \mathrm{~K}$ after hydrogen charging, which is attributed to hydrogen desorption from vacancies and small vacancy clusters (see inset of Figure 1a). These observations are in line with Fukai's report [37] of superabundant vacancy formation and agglomeration during high pressure and high temperature hydrogen exposure of FCC metals.

A long-lifetime, $\tau_{2}$, component is also detected for Ni-201 specimens that were strained, but not hydrogen charged (Figure 3b, 3c, and Table I). These lifetime values indicate that plastic deformation promotes vacancy formation and coalescence. Such vacancy formation phenomena 
have been well-documented in literature; Zehetbauer provides a review of strain-induced vacancy formation mechanisms [38]. Positrons lifetimes do not depend on measurement duration for this condition because the defect structures are more stable during deformation than those in the presence of hydrogen.

Lastly, the process of hydrogen charging followed by plastic deformation leads to an increase in the $\tau_{2}$ component values when compared with the values for either hydrogen charging or straining alone. The relative intensity of the long lifetime, $I_{2}$, in strained Ni-201 alloy samples increases from about $25 \%$ for non-charged samples to about $30 \%$ in the presence of hydrogen indicating that hydrogen promotes clustering of strain-induced vacancies and vacancy complexes. The slight increase of $\tau_{2}$ and $I_{2}$ with PAS measurement time for the polycrystalline alloys may also be evidence that the grain boundaries of the hydrogen-charged + strained samples are far from equilibrium, consuming the excessive vacancies generated during hydrogen charging/deformation, thereby providing additional free volume. In addition to altering PAS parameters, straining of the hydrogen-charged samples also increases the temperature position of the minor TDS peak in Ni-201 samples to approximately $770 \mathrm{~K}$ (see Figure 1b). The shift may originate from hydrogen trapping in the larger vacancy clusters induced by plastic straining. However, the effect of vacancy cluster size on hydrogen trapping energy, which defines the TDS peak position, is still unknown.

Elevated monovacancy and vacancy cluster concentrations in the hydrogen-charged and strained condition are consistent with the HESIV model proposed for pure Fe and steel $[7,12,16]$. Hatano et al. [21] found similar positron lifetime trends in hydrogen-charged austenitic stainless steels strained to failure in tension; they suggest that the appearance and increase in $\tau_{2}$ beyond the lifetime for monovacancies in austenite corresponds with hydrogen-enhanced vacancy clustering.

The effects of hydrogen charging, straining of non-charged specimens, and straining of hydrogen-charged specimens on vacancy cluster formation are consistent with previous studies. In the present work, two additional variables, namely, grain size and deformation temperature, were emphasized. When considering the role of grain size on the interaction between hydrogen and various lattice defects, it is necessary to account for the spatial distribution of vacancies and vacancy clusters within the material. It is assumed here that hydrogen charging enhances generation of thermally activated vacancies primarily at grain boundaries and specimen free 
surfaces. Using the average time for a vacancy diffusion hop in $\mathrm{Ni}$ at $600 \mathrm{~K}\left(0.6 \times 10^{-4} \mathrm{~s}\right)$ [35] the upper value of the characteristic diffusion path for a monovacancy under the applied charging conditions is estimated to be $33 \mu \mathrm{m}$. This implies the SC and 1-mm grain size samples will develop an approximately 30- $\mu \mathrm{m}$ thick mantle-like layer of vacancies, and their associated complexes, adjacent to free surfaces and grain boundaries. Conversely, hydrogen charging of 35$\mu \mathrm{m}$ grain size Ni-201 alloy specimens results in a nearly homogeneous distribution of hydrogen, vacancies, and vacancy-hydrogen complexes over the grain bulk. Additionally, specimen cooling to room temperature, immediately after hydrogen charging, has only a minor effect on the vacancy and vacancy complex distribution, but the resulting hydrogen distribution over the sample cross-section becomes macroscopically non-homogeneous.

Grain size effects on vacancy generation and clustering are assessed as a function of material condition. In the case of samples that were only hydrogen charged, monovacancies dominate the defect structure regardless of the grain size; the ratio of $I_{1}: I_{2}$ ranges from $3: 1$ to $7: 1$ (see Table I). However, decreasing grain size (increasing grain boundary area) does increase the propensity for vacancy clustering; $I_{2}$ values for the SC and coarse-grained Ni-201 alloy are about $15 \%$, while for the fine-grained Ni-201 alloy $I_{2}$ increases to $25 \%$. Similar $I_{2}$ values are measured for samples deformed without hydrogen. However, both $\tau_{2}$ and $I_{2}$ values increase further when samples are deformed in the presence of hydrogen (see Table I), revealing that hydrogen charging enhances vacancy generation and clustering over and above the defect structures generated during plastic deformation. Since the grain boundaries always contain free volume, even in annealed polycrystalline materials, it is plausible that plastic accommodation during the course of straining results in hydrogen-enhanced free volume generation in grain boundaries or volumes adjacent to them. Again, TDS supports the PAS data. The minor TDS peak observed at $750 \mathrm{~K}$ after hydrogen charging shifts to $770 \mathrm{~K}$ for polycrystalline samples that were strained after hydrogen charging. Because no minor peak was found in the hydrogencharged and strained Ni SC sample it is surmised that growing vacancy complexes are situated at the grain boundaries and/or volumes adjacent to them.

The effect of deformation temperature on vacancy-hydrogen interactions is less straightforward. The $\tau_{2}$ values for Ni-201 alloy deformed at $77 \mathrm{~K}$, both without and with hydrogen are consistently lower than those for samples deformed at $293 \mathrm{~K}$, irrespective of grain size. This suggests that temperature limits the maximum attainable cluster size. Additionally, the 
$\tau_{2}$ values are higher for the hydrogen-charged Ni-201 alloy, compared with the non-charged material for both deformation temperatures, again evidencing that hydrogen enhances vacancy cluster agglomeration beyond that attainable by plastic deformation alone.

The thermodynamics of vacancy formation and clustering can be assessed in terms of literature models. Specifically, Kirchheim's defactant concept suggests that hydrogen may amplify vacancy concentrations by lowering the vacancy formation energy, thereby producing the observed changes in vacancy size with changing deformation temperature. This model considers hydrogen a 'defactant' (defect acting agent) in the microstructure that modifies the thermodynamic driving force for defect generation and stability [39,40]. The vacancy formation energy, $E_{V}$, is lowered when $Z$ excess solute hydrogen atoms segregate to a formation site. The overall vacancy formation energy is therefore: $E_{V}^{H}=E_{V}^{0}-Z \mu_{H}$, where $E_{V}^{H}$ is the hydrogen modified vacancy formation energy, $E_{V}^{0}$ is the nucleation energy for formation of stable vacancies without hydrogen, and $\mu_{H}$ is the chemical potential of hydrogen [40]. During deformation at $293 \mathrm{~K}$, the diffusion-mobile hydrogen reduces the $E_{V}^{0}$ to $E_{V}^{H}$ and stabilizes the strain-induced vacancies. Conversely, during deformation at $77 \mathrm{~K}$, when hydrogen diffusion is suppressed, the strain-induced vacancies and vacancy complexes may be expected to form in fashion similar to those in non-charged Ni-201 alloys. However, Figures $3 \mathrm{~b}$ and $3 \mathrm{c}$ reveal an unexpected enhancement in $\tau_{2}$ values for hydrogen-charged specimens strained at $77 \mathrm{~K}$, compared to similar non-charged specimens. This implies that the vacancy agglomeration mechanism at $77 \mathrm{~K}$ may radically differ from that operating at $293 \mathrm{~K}$. However, the mechanism driving the low-temperature free-volume generation is unclear.

Finally, hydrogen-affected plastic flow behavior shown in Figure 4 can be interpreted in the context of two temperature-dependent mechanisms: cross-slip and 'Orowan type' hardening. It is important to first note that in FCC alloys the collective motion of dislocations governs plastic flow properties. At low strains, when there are few mobile dislocations, hardening may be dominated by solute drag [16,41], but this mechanism will soon be overwhelmed by dislocation multiplication with increasing strain. The presence of hydrogen in solid solution in the Ni lattice increases the propensity for dislocation multiplication, rather than new source activation, by decreasing the critical stress required for Frank-Read source activation and loop expansion [41]. In a deforming crystal, this amounts to increased flow localization. Therefore, the yield strength 
and work hardening increases shown in Figure 4 and Table II must be governed by hydrogen interacting with the evolving defect structure in a temperature dependent manner.

At $77 \mathrm{~K}$, where hydrogen is essentially immobile in the Ni lattice $\left(D_{H, 77 \mathrm{~K}} \sim 10^{-31} \mathrm{~cm}^{2} \mathrm{~s}^{-1}\right)$, strengthening mechanisms requiring the diffusive movement of hydrogen will be limited. However, it is possible that large vacancy complexes (which may collapse to form prismatic loops) induce a long-distance elastic strain field leading to an 'Orowan type' interaction (e.g. dispersion-like hardening) with dislocations, similar to the barrier strengthening induced by Frank loops generated during neutron irradiation [42]. These hydrogen-enhanced barriers to dislocation motion are especially effective at $77 \mathrm{~K}$ when cross-slip is also inhibited by temperature, leading to a larger hydrogen-induced yield strength increase than during room temperature deformation (see Table II). Interpreting the flow properties of hydrogen-charged Ni strained at room temperature must also consider the effects of mobile hydrogen $\left(D_{H, 300 \mathrm{~K}} \sim 10^{-10}\right.$ $\mathrm{cm}^{2} \mathrm{~s}^{-1}$ ), i.e. the interaction of diffusion-mobile solute hydrogen with dislocations [16], particularly dissociated partial dislocations. As described by Delafosse [41], hydrogen screening of dislocation pair interactions decreases the repulsion between partials of a dissociated screw dislocation thereby increasing the work for partial recombination and limiting the probability of dislocation cross-slip. Plastic flow behavior is also impacted by the hydrogen-affected evolution of dislocation structures during deformation. Delafosse notes that Ni single crystals oriented for single glide develop equiaxed dislocation cells, but hydrogen charging leads to the formation of a planar structure composed of dislocation walls, and thus a smaller mean free path [41], when assessed at the same increment of strain. Planar dislocation walls are characteristic of materials with a low probability for cross-slip (and often a low stacking fault energy) [41], preventing dynamic recovery and cell formation. Consistent with these previous studies, the hydrogenenhanced hardening observed during room temperature deformation in the present study is likely dominated by cross-slip restriction, driven by a decrease in the dislocation mean free path.

\section{Conclusions}

Mechanical testing combined with positron annihilation spectroscopy and thermal desorption spectroscopy reveal important aspects of the role of alloy grain size and deformation temperature on hydrogen-enhanced vacancies and clustering. In particular, it was found that: 
1) Hydrogen charging of coarse-grained and fine-grained $\mathrm{Ni}-201$ and $\mathrm{Ni} \mathrm{SC}$ enhanced vacancy formation. Plastic deformation of hydrogen-charged $\mathrm{Ni}$ alloys resulted in the generation of additional free volume in the form of vacancy clusters.

2) During the course of long-term PAS measurement, the positron lifetime of hydrogencharged and strained Ni-201 alloys increase. No time evolution was observed for the $\mathrm{Ni}$ $\mathrm{SC}$, indicating that grain boundaries play a specific role in hydrogen-enhanced vacancy agglomeration.

3) Hydrogen-enhanced vacancy cluster density is inversely proportional to grain size, as indicated by an increase in positron lifetime intensity with decreasing grain size. Similarly, positron lifetime values increase with decreasing deformation temperature, That is, temperature limits the maximum attainable cluster size with increasing cluster density.

4) A secondary thermal desorption peak is detected for hydrogen-charged and hydrogencharged + strained Ni-201 alloy samples, but not for SC samples, further suggesting that vacancies agglomerate on grain boundaries and in the adjacent volumes.

5) Hydrogen charging increases both the yield strength and work hardening rate of Ni-201 and Ni SC. The increases are temperature dependent, suggesting that hydrogen-enhanced vacancy clustering may induce an 'Orowan type' hardening component, which is effective even when hydrogen is essentially immobile; mobile hydrogen provides an additional hardening increase by interacting with mobile dislocations to restrict dislocation cross-slip.

This work confirms that hydrogen enhances free volume formation and agglomeration, especially at or adjacent to grain boundaries, and points to the importance of temperature in developing a thermodynamic framework for a vacancy-enhanced hydrogen degradation mechanism. However, an important question remains to be answered: do heightened vacancy concentrations during deformation in the presence of hydrogen also contribute to the propensity for intergranular failure of $\mathrm{Ni}$ alloys?

\section{Acknowledgement}

This work was supported by S.K. Lawrence's DOE NNSA Stewardship Science Graduate Fellowship [grant DE-FC52-08NA28752] and the Laboratory Directed Research and 
Development program [grant SNL-LDRD-173116] at Sandia National Laboratories, a multiprogram laboratory managed and operated by Sandia Corporation, a wholly owned subsidiary of Lockheed Martin Corporation, for the U.S. Department of Energy's National Nuclear Security Administration under contract DE-AC04-94AL85000. Y. Yagodzinsky and H. Hänninen extend appreciation to the Academy of Finland for support of the study in the frame of HYMESS project. 


\section{REFERENCES}

[1] W. Gerberich, Modeling hydrogen induced damage mechanisms in metals, in: R.P. Gangloff, B.P. Somerday (Eds.), Gaseous Hydrog. Embrittlement Mater. Energy Technol. Vol. 2 Mech. Model., Futur. Dev., Woodhead, Philadelphia, 2012: pp. 209-246.

[2] S.P. Lynch, Mechanisms of environmentally assisted cracking in Al-Zn-Mg single crystals, Corros. Sci. 22 (1982) 925-937.

[3] H.K. Birnbaum, P. Sofronis, Hydrogen-enhanced localized plasticity - a mechanism for hydrogen-related fracture, Mater. Sci. Eng. A. 176 (1994) 191-202.

[4] R. Oriani, P. Joshephic, Equilibrium aspects of hydrogen induced cracking in steels, Acta Metall. 22 (1974) 1065-1075.

[5] Y. Jagodzinski, H. Hanninen, O. Tarasenko, S. Smuk, Interaction of hydrogen with dislocation pile-ups and hydrogen induced softening of pure iron, Scr. Mater. 43 (2000) 245-251.

[6] W.W. Gerberich, Y.T. Chen, Hydrogen-controlled cracking-An approach to threshold stress intensity, Metall. Trans. A. 6 (1975) 271-278.

[7] J. Kameda, C. McMahon, Solute segregation and brittle fracture in an alloy steel, Metall. Mater. Trans. A. 11 (1980) 91-101.

[8] A. Kimura, H.K. Birnbaum, Hydrogen induced grain boundary fracture in high purity nickel and its alloys - Enhanced hydrogen diffusion along grain boundaries, Acta Metall. 36 (1988) 757-766.

[9] M. Nagumo, Hydrogen related failure of steels - a new aspect, Mater. Sci. Technol. 20 (2004) 940-950.

[10] Y. Fukai, N. Okuma, Formation of superabundant vacancies in Pd hydride under high hydrogen pressures, Phys. Rev. Lett. 73 (1994) 1640-1643.

[11] H. Osono, T. Kino, Y. Kurokawa, Y. Fukai, Agglomeration of hydrogen-induced vacancies in nickel, J. Alloys Compd. 231 (1995) 41-45.

[12] M. Nagumo, K. Ohta, H. Saitoh, Deformation induced defects in iron revealed by thermal desorption spectroscopy of tritium, Scr. Mater. 40 (1999) 313-319.

[13] K. Sakaki, T. Kawase, M. Hirato, M. Mizuno, H. Araki, Y. Shirai, M. Nagumo, The effect of hydrogen on vacancy generation in iron by plastic deformation, Scr. Mater. 55 (2006) 1031-1034.

[14] M. Nagumo, T. Ishikawa, T. Endoh, Y. Inoue, Amorphization associated with crack propagation in hydrogen-charged steel, Scr. Mater. 49 (2003) 837-842.

[15] M. Nagumo, M. Nakamura, K. Takai, Hydrogen thermal desorption relevant to selayedfracture susceptibility of high-strength steels, Metall. Mater. Trans. A. 32 (2001) 339-347.

[16] T. Boniszewski, G.C. Smith, The influence of hydrogen on the plastic deformation, ductility, and fracture of nickel in tension, Acta Metall. 11 (1963) 165-178.

[17] A.H. Windle, G.C. Smith, The effect of hydrogen on the plastic deformation of nickel 
single crystals, Met. Sci. J. 2 (1968) 187-191.

[18] H. Fuchigami, H. Minami, M. Nagumo, Effect of grain size on the susceptibility of martensitic steel to hydrogen-related failure, Philos. Mag. Lett. 86 (2006) 21-29.

[19] K. Takai, H. Shoda, H. Suzuki, M. Nagumo, Lattice defects dominating hydrogen-related failure of metals, Acta Mater. 56 (2008) 5158-5167.

[20] H. Ohkubo, Z. Tang, Y. Nagai, M. Hasegawa, T. Tawara, M. Kiritani, Positron annihilation study of vacancy-type defects in high-speed deformed $\mathrm{Ni}, \mathrm{Cu}$ and $\mathrm{Fe}$, Mater. Sci. Eng. A. 350 (2003) 95-101.

[21] M. Hatano, M. Fujinami, K. Arai, H. Fujii, M. Nagumo, Hydrogen embrittlement of austenitic stainless steels revealed by deformation microstructures and strain-induced creation of vacancies, Acta Mater. 67 (2014) 342-353.

[22] P. Hautojarvi, ed., Positrons in Solids, Topics in Current Physics, vol. 12, Springer, Heidelberg, 1979.

[23] F. Tuomisto, I. Makkonen, Defect identification in semiconductors with positron annihilation: Experiment and theory, Rev. Mod. Phys. 85 (2013) 1583-1631.

[24] H. Rajainmaki, S. Linderoth, H.E. Hansen, R.M. Nieminen, Defect recovery and hydrogen-vacancy interactions in nickel between 20 and 650 K, J. Phys. F Met. Phys. 18 (1988) 1109-1118.

[25] B.L. Shivachev, T. Troev, T. Yohiie, Positron lifetime computations of defects in nickel containing hydrogen or helium, J. Nucl. Mater. 306 (2002) 105-111.

[26] G. Dlubek, O. Brümmer, E. Hensel, Positron annihilation investigation for an estimation of the dislocation density and vacancy concentration of plastically deformed polycrystalline Ni of different purity, Phys. Status Solidi. 34 (1976) 737-746.

[27] E. Kuramoto, H. Abe, M. Takenaka, F. Hori, Y. Kamimura, M. Kimura, K. Ueno, Positron annihilation lifetime study of irradiated and deformed Fe and Ni, J. Nucl. Mater. 239 (1996) 54-60.

[28] D.H. Lassila, H.K. Birnbaum, Intergranular fracture of nickel: the effect of hydrogensulfur co-segregation, Acta Met. 35 (1987) 1515-1822.

[29] S.M. Bruemmer, R.H. Jones, M.T. Thomas, D.R. Baer, Influence of sulfur, phosphorus , and antimony segregation on the intergranular hydrogen embrittlement of nickel, Metall. Mater. Trans. A. 14 (1983) 223-232.

[30] W.C. Johnson, J.E. Doherty, B.H. Kear, A.F. Giamei, Confirmation of sulfur embrittlement in nickel alloys, Scr. Metall. 8 (1974) 971-974.

[31] O. Todoshchenko, Y. Yagodzinskyy, H. Hänninen, Thermal desorption of hydrogen from AISI 316L stainless steel and pure nickel, Defect Diffus. Forum. 344 (2013) 71-77.

[32] K.G. Lynn, R. Ure, J.G. Byrne, The effect of plastic deformation on positron annihilation in copper of varying grain size, Acta Metall. 22 (1974) 1075-1077.

[33] N.M. Nancheva, K. Saarinen, Positron lifetime studies of shock loaded nickel, Scr. Metall. 
20 (1986) 1085-1088.

[34] M.J. Puska, R.. Nieminen, Defect spectroscopy with positrons : a general calculational method, J. Phys. F Met. Phys. 13 (1983) 333-346.

[35] Y. Wang, D. Connétable, D. Tanguy, Hydrogen influence on diffusion in nickel from first-principles calculations, Phys. Rev. B. 91 (2015) 1-9.

[36] D. Tanguy, Y. Wang, D. Connétable, Stability of vacancy-hydrogen clusters in nickel from first-principles calculations, Acta Mater. 78 (2014) 135-143.

[37] Y. Fukai, Superabundant vacancies formed in metal-hydrogen alloys, Phys. Scr. T103 (2003) 11-14.

[38] M. Zehetbauer, Effects of Non-equilibrium Vacancies on Strengthening, Key Eng. Mater. 97 (1994) 287-306.

[39] R. Kirchheim, Reducing grain boundary, dislocation line and vacancy formation energies by solute segregation I. Theoretical background, Acta Mater. 55 (2007) 5129-5138.

[40] R. Kirchheim, Reducing grain boundary, dislocation line and vacancy formation energies by solute segregation II. Experimental evidence and consequences, Acta Mater. 55 (2007) 5139-5148.

[41] D. Delafosse, Hydrogen effects on the plasticity of face centered cubic (fcc) crystals, in: R.P. Gangloff, B.P. Somerday (Eds.), Gaseous Hydrog. Embrittlement Mater. Energy Technol. Vol. 2 Mech. Model. , Futur. Dev., Woodhead, Philadelphia, 2012: pp. 247-285.

[42] N. Hashimoto, T. Byun, K. Farrell, Microstructural analysis of deformation in neutronirradiated fcc materials, J. Nucl. Mater. 351 (2006) 295-302. 


\section{TABLES:}

Table I. Average lifetime $\tau_{\text {ave }}$, short lifetime $\tau_{1}$, long-term lifetime $\tau_{2}$ and its relative intensity $I_{2}$ for positron annihilation in nickel single crystal and both studied Ni-201 alloys after different treatments.

\begin{tabular}{|c|c|c|c|c|c|}
\hline Sample Family & Condition & $\tau_{\text {ave, }}$ ps & $\tau_{1}, \mathrm{ps}$ & $\tau_{2}, \mathrm{ps}$ & $\mathrm{I}_{2}, \%$ \\
\hline \multirow{4}{*}{$\overline{S C}$} & As-supplied & 128.2 & $116 \pm 1$ & $240 \pm 4$ & $9.9 \pm 0.6$ \\
\hline & $\mathrm{H}_{2}$ charged & 136.6 & $126 \pm 2$ & $219 \pm 11$ & $13.5 \pm 3$ \\
\hline & Strained $293 \mathrm{~K}$ & 147.7 & $125 \pm 3$ & $221 \pm 6$ & $23.6 \pm 3$ \\
\hline & $\begin{array}{l}\mathrm{H}_{2} \text { charged }+ \\
\text { Strained } 293 \mathrm{~K}\end{array}$ & 172.9 & $132 \pm 2$ & $280 \pm 4$ & $27.9 \pm 1$ \\
\hline \multirow[t]{6}{*}{ 1-mm grain size } & As-annealed & 145.1 & $121 \pm 3$ & $218 \pm 9$ & $25 \pm 4$ \\
\hline & $\mathrm{H}_{2}$ charged & 152.2 & $134 \pm 3$ & $255 \pm 14$ & $15 \pm 3$ \\
\hline & Strained $293 \mathrm{~K}$ & 202.9 & $161 \pm 3$ & $303 \pm 6$ & $29 \pm 2$ \\
\hline & Strained $77 \mathrm{~K}$ & 149.6 & $162 \pm 5$ & $247 \pm 17$ & $22 \pm 8$ \\
\hline & $\begin{array}{l}\mathrm{H}_{2} \text { charged }+ \\
\text { strained } 293 \mathrm{~K}\end{array}$ & 203.7 & $163 \pm 4$ & $308 \pm 10$ & $28 \pm 4$ \\
\hline & $\begin{array}{l}\mathrm{H}_{2} \text { charged }+ \\
\text { strained } 77 \mathrm{~K}\end{array}$ & 213.7 & $170 \pm 6$ & $307 \pm 11$ & $32 \pm 5$ \\
\hline \multirow[t]{6}{*}{ 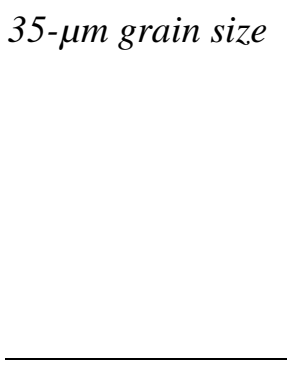 } & As-supplied & 148.0 & $120 \pm 3$ & $217 \pm 6$ & $33 \pm 4$ \\
\hline & $\mathrm{H}_{2}$ charged & 156.1 & $136 \pm 4$ & $216 \pm 11$ & $25 \pm 7$ \\
\hline & Strained $293 \mathrm{~K}$ & 195.5 & $166 \pm 3$ & $298 \pm 8$ & $22 \pm 3$ \\
\hline & Strained $77 \mathrm{~K}$ & 196.6 & $166 \pm 4$ & $284 \pm 9$ & $26 \pm 4$ \\
\hline & $\begin{array}{l}\mathrm{H}_{2} \text { charged }+ \\
\text { strained } 293 \mathrm{~K}\end{array}$ & 209.6 & $166 \pm 3$ & $314 \pm 6$ & $29 \pm 2$ \\
\hline & $\begin{array}{l}\mathrm{H}_{2} \text { charged }+ \\
\text { strained } 77 \mathrm{~K}\end{array}$ & 212.7 & $167 \pm 3$ & $305 \pm 3$ & $31 \pm 2$ \\
\hline
\end{tabular}

Table II. Percent increase in yield strength $(Y S)$ and work hardening $(W H)$ values between the non-charged and hydrogen-charged conditions. Work hardening rates are calculated at $6 \%$ and $8 \%$ strain.

\begin{tabular}{|c|c|c|c|}
\hline Sample & $Y S$ & $W H @ 6 \%$ & $W H @ 8 \%$ \\
\hline $\mathrm{SC}[001], 293 \mathrm{~K}$ & 42 & 48 & 55 \\
\hline $1 \mathrm{~mm}, 293 \mathrm{~K}$ & 5 & 56 & 86 \\
\hline 1 mm, $77 \mathrm{~K}$ & 19 & 7 & 29 \\
\hline $35 \mu \mathrm{m}, 293 \mathrm{~K}$ & 8 & 58 & 69 \\
\hline $35 \mu \mathrm{m}, 77 \mathrm{~K}$ & 52 & 16 & 12 \\
\hline
\end{tabular}




\section{FIGURE CAPTIONS}

Figure 1. Hydrogen desorption flux for (a) hydrogen-charged Ni SC reference material, 1-mm grain size material, and 35- $\mu \mathrm{m}$ grain size material and (b) SC strained at $293 \mathrm{~K}$ after hydrogen charging, 1-mm and 35- $\mu \mathrm{m}$ materials strained at $77 \mathrm{~K}$ and $293 \mathrm{~K}$ after hydrogen charging. Sample thicknesses, $h$, are tabulated for each specimen.

Figure 2. Positron lifetime spectra in as-received and processed 5N Ni single crystal samples. The two-component nature of the spectra is evident, as well as the different intensities and values of the second lifetime component.

Figure 3. Time evolution of (a) $\tau_{\text {ave }}$ for $\mathrm{SC} \mathrm{Ni}$, coarse-grained $\mathrm{Ni}-201$, fine-grained $\mathrm{Ni}-201$, (b) $\tau_{2}$ and $I_{2}$ for fine-grained Ni-201, and (c) $\tau_{2}$ and $I_{2}$ for coarse-grained Ni-201. For clarity only data for samples deformed at $77 \mathrm{~K}$ are presented.

Figure 4. Engineering stress-strain curves for SC, 1-mm grain size, and 35- $\mu \mathrm{m}$ and grain size Ni201 deformed in tension at (a) $293 \mathrm{~K}$ and (b) $77 \mathrm{~K}$, with and without hydrogen. 


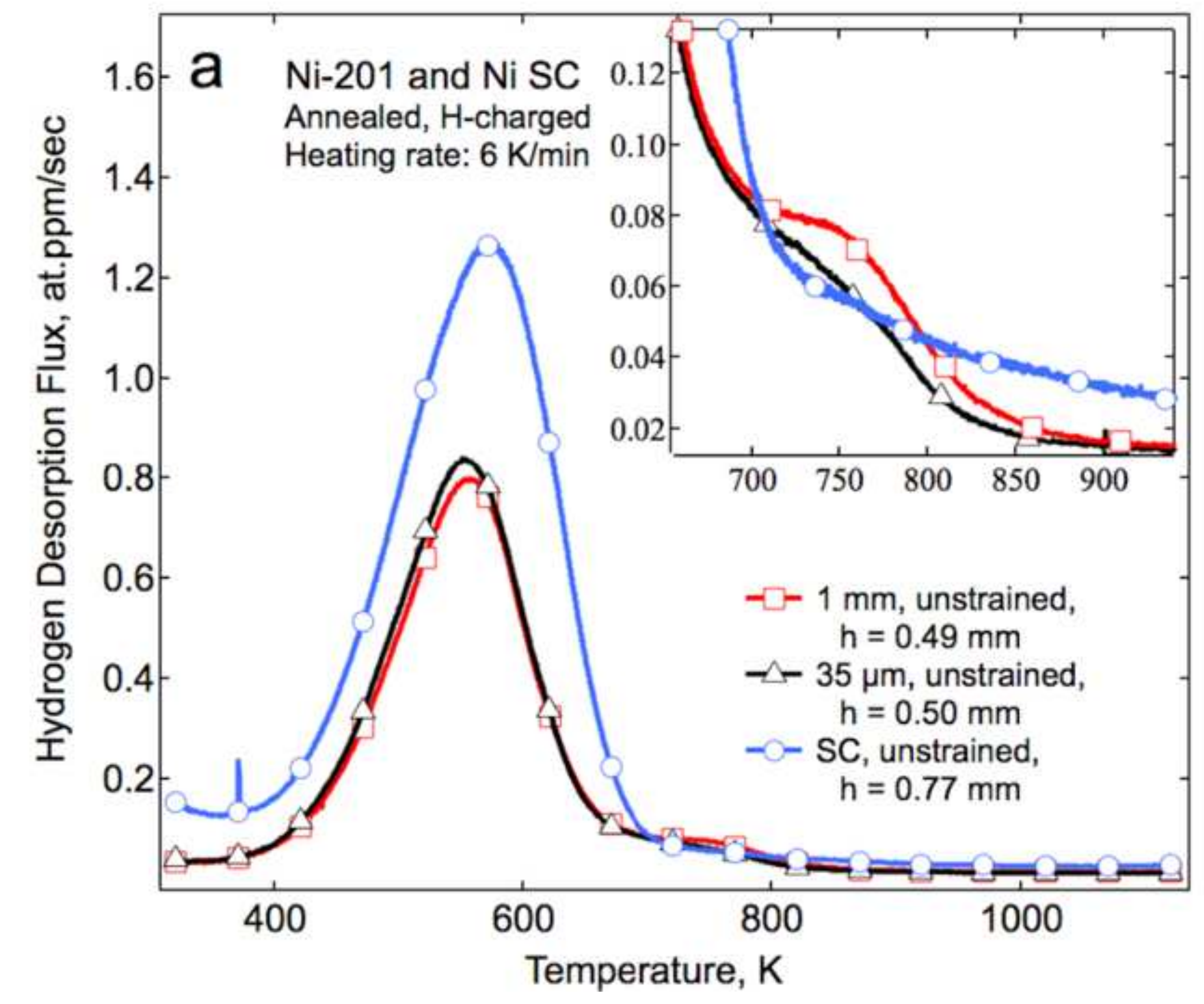




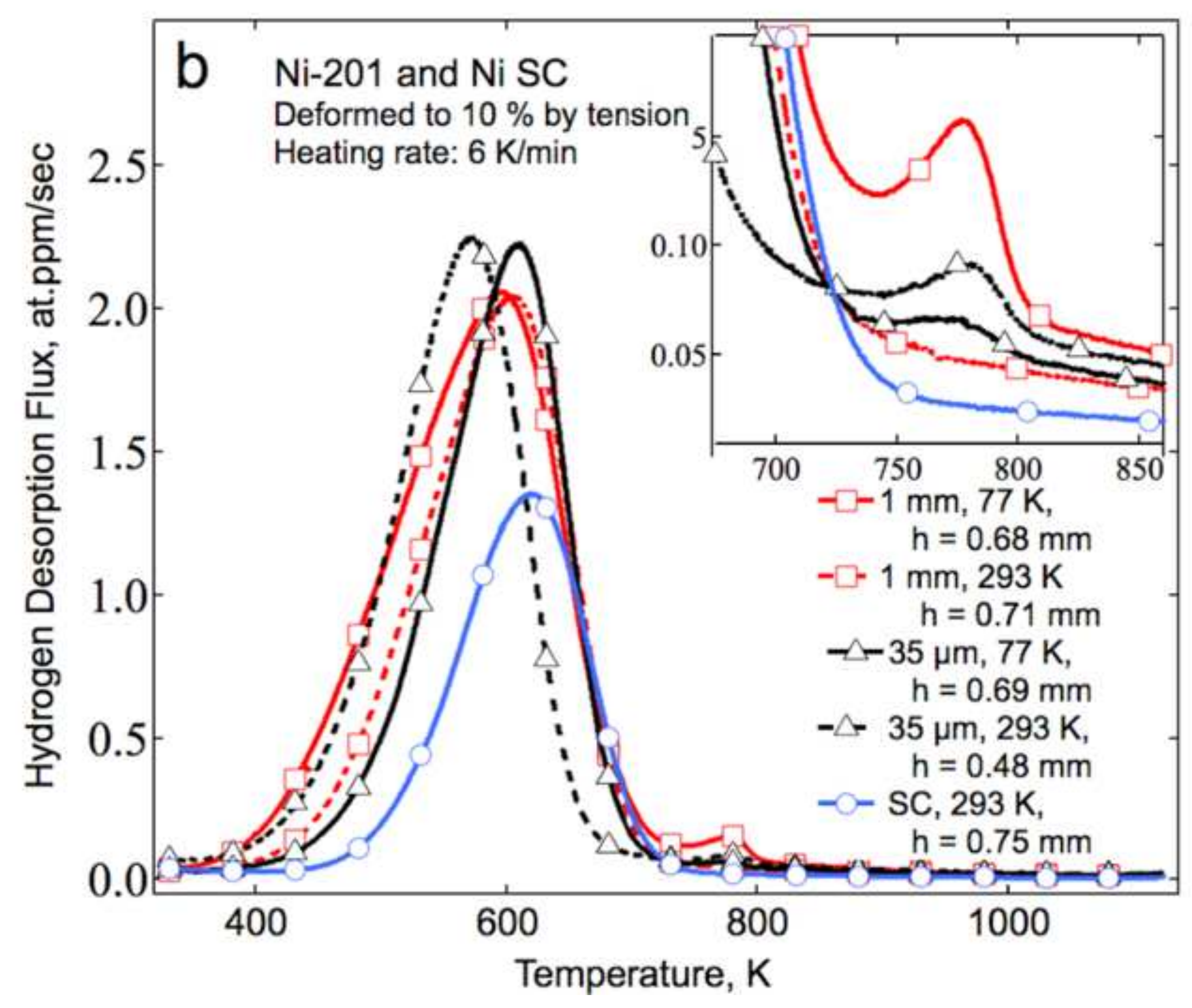




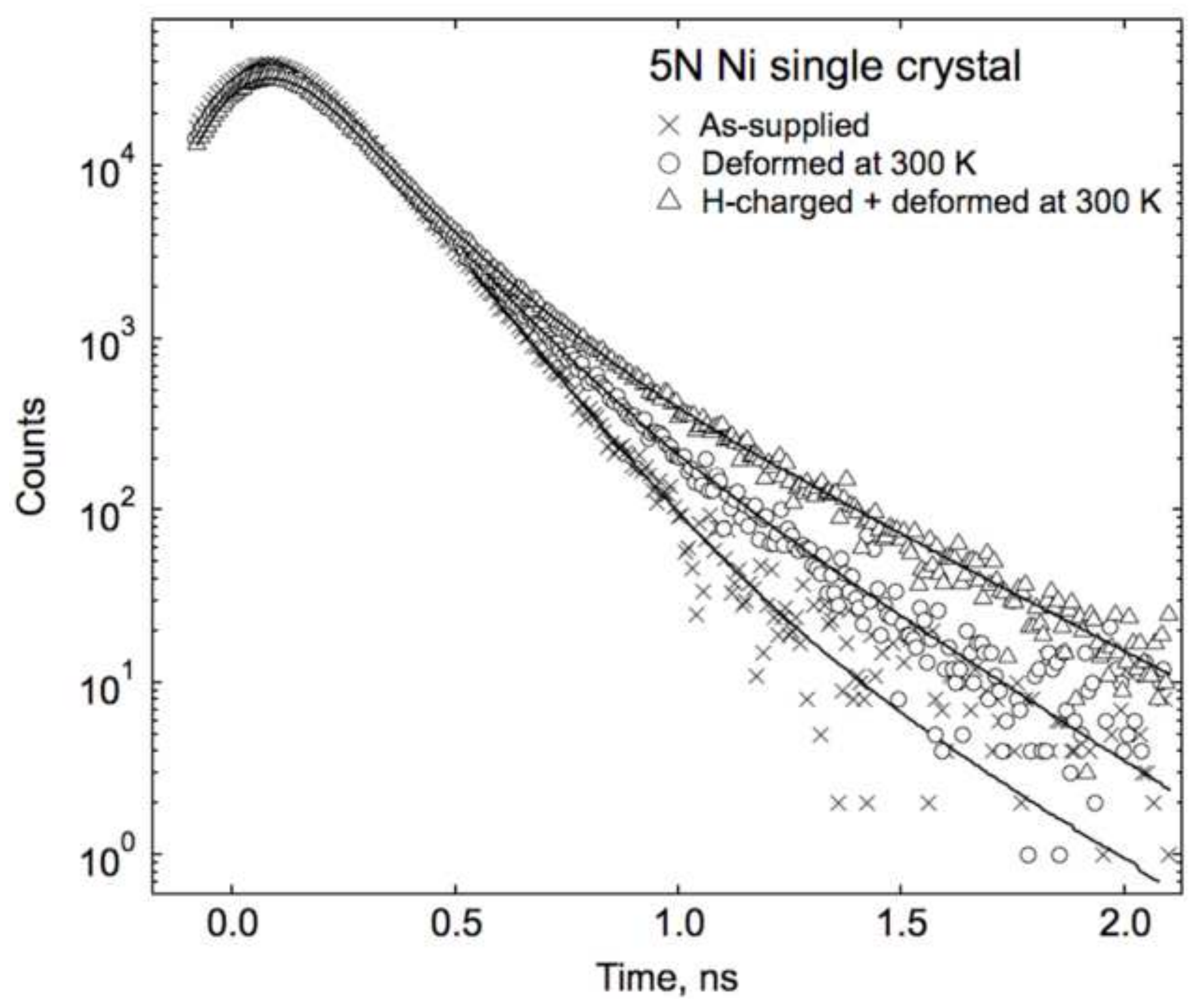




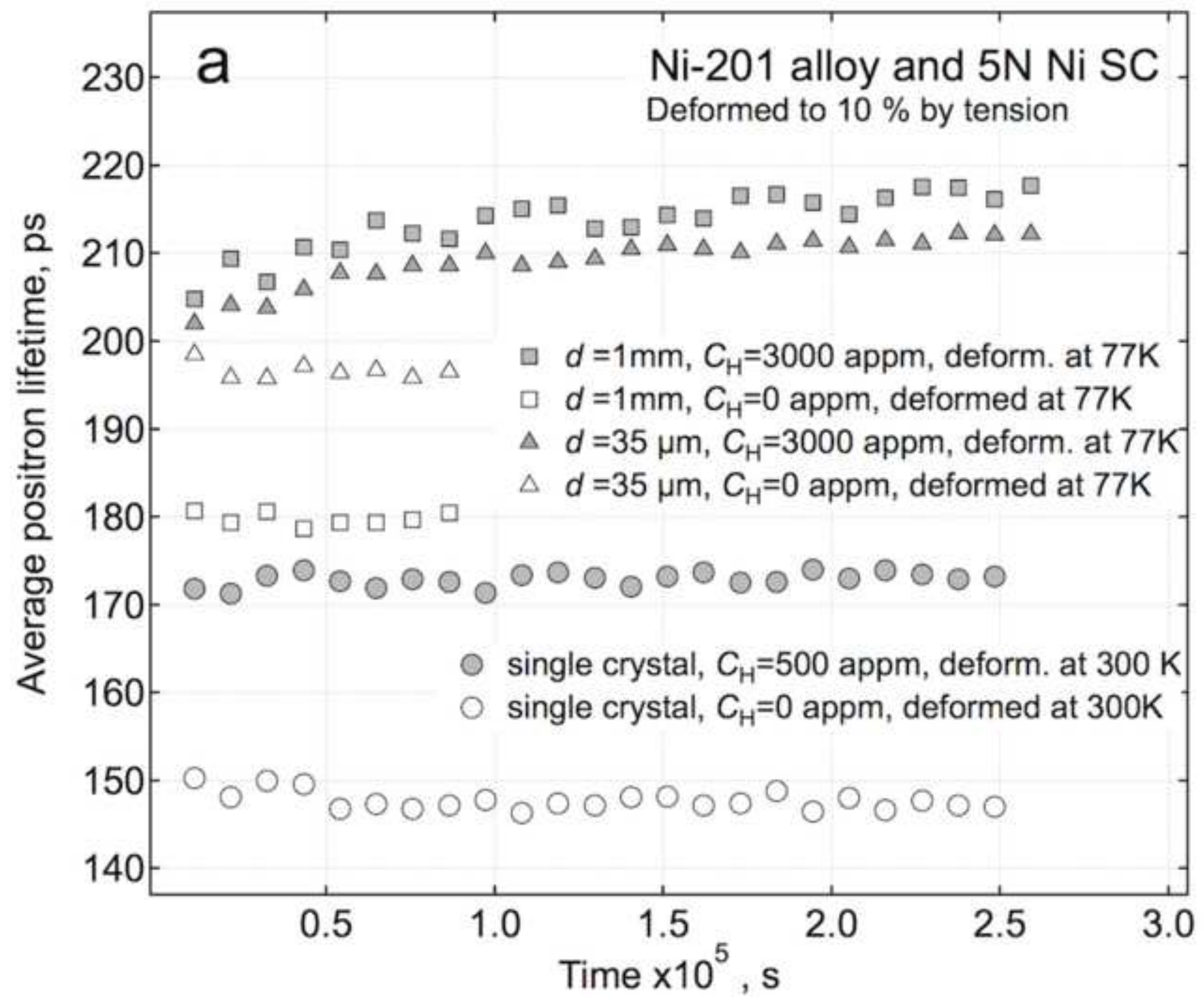




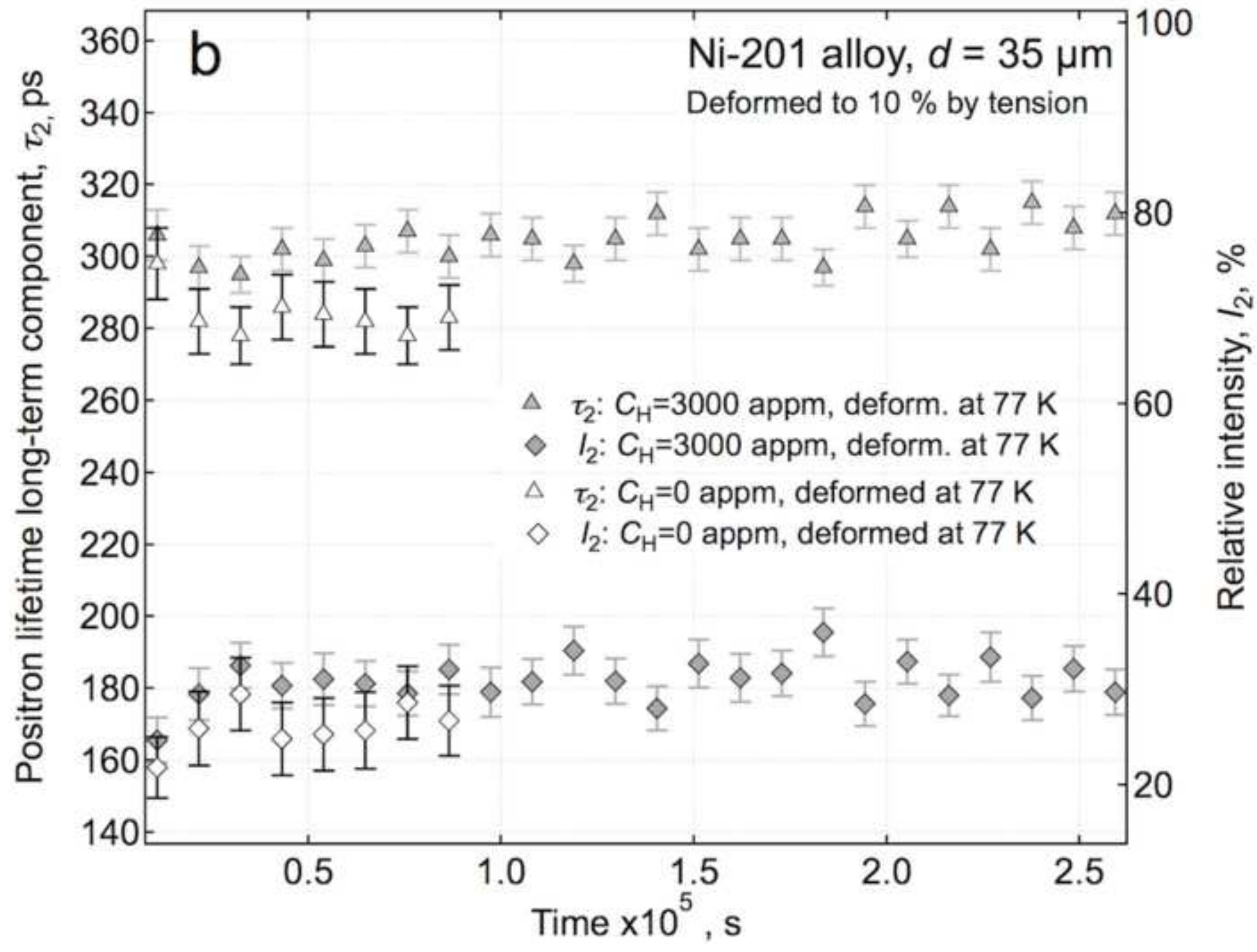




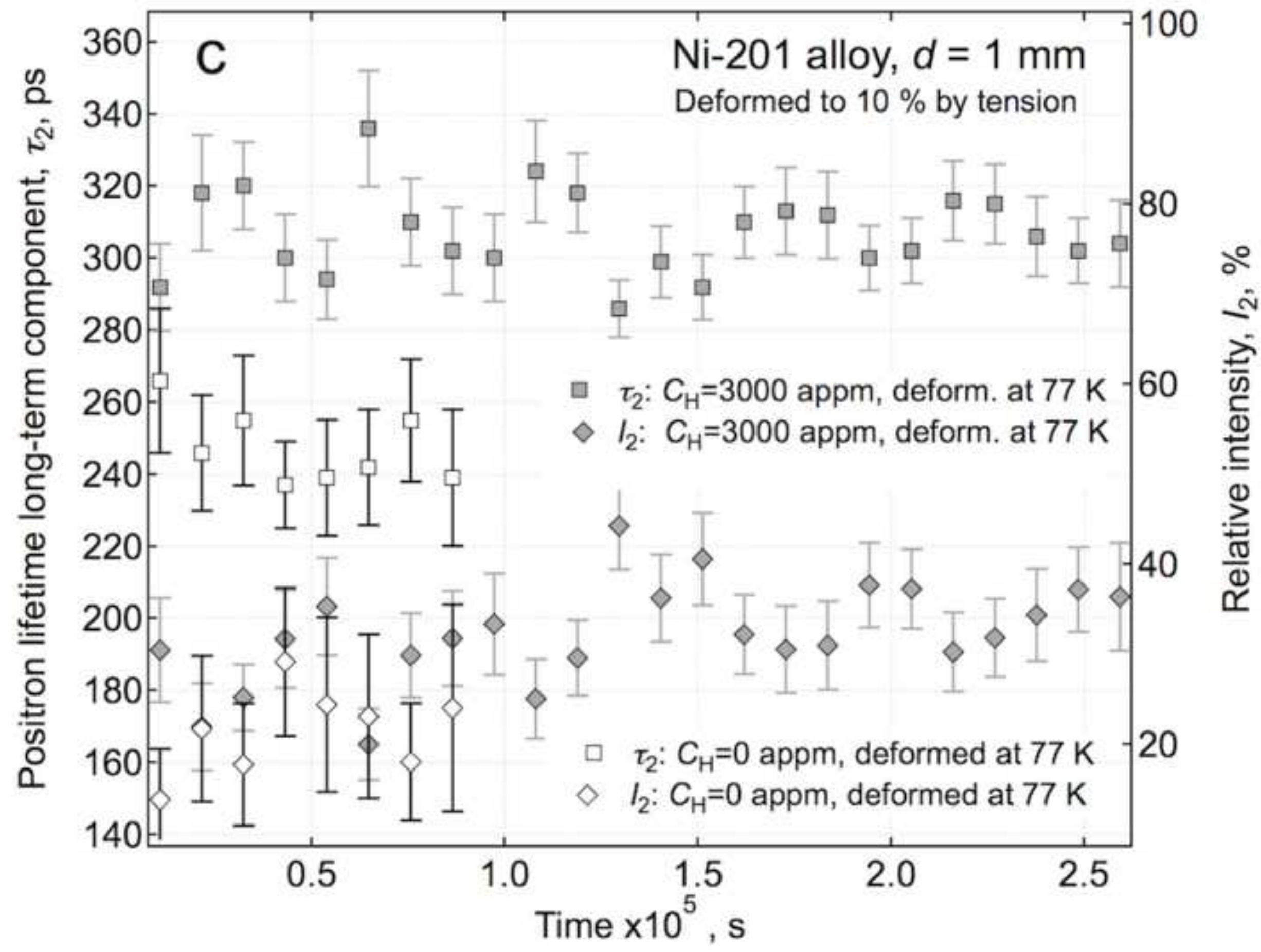




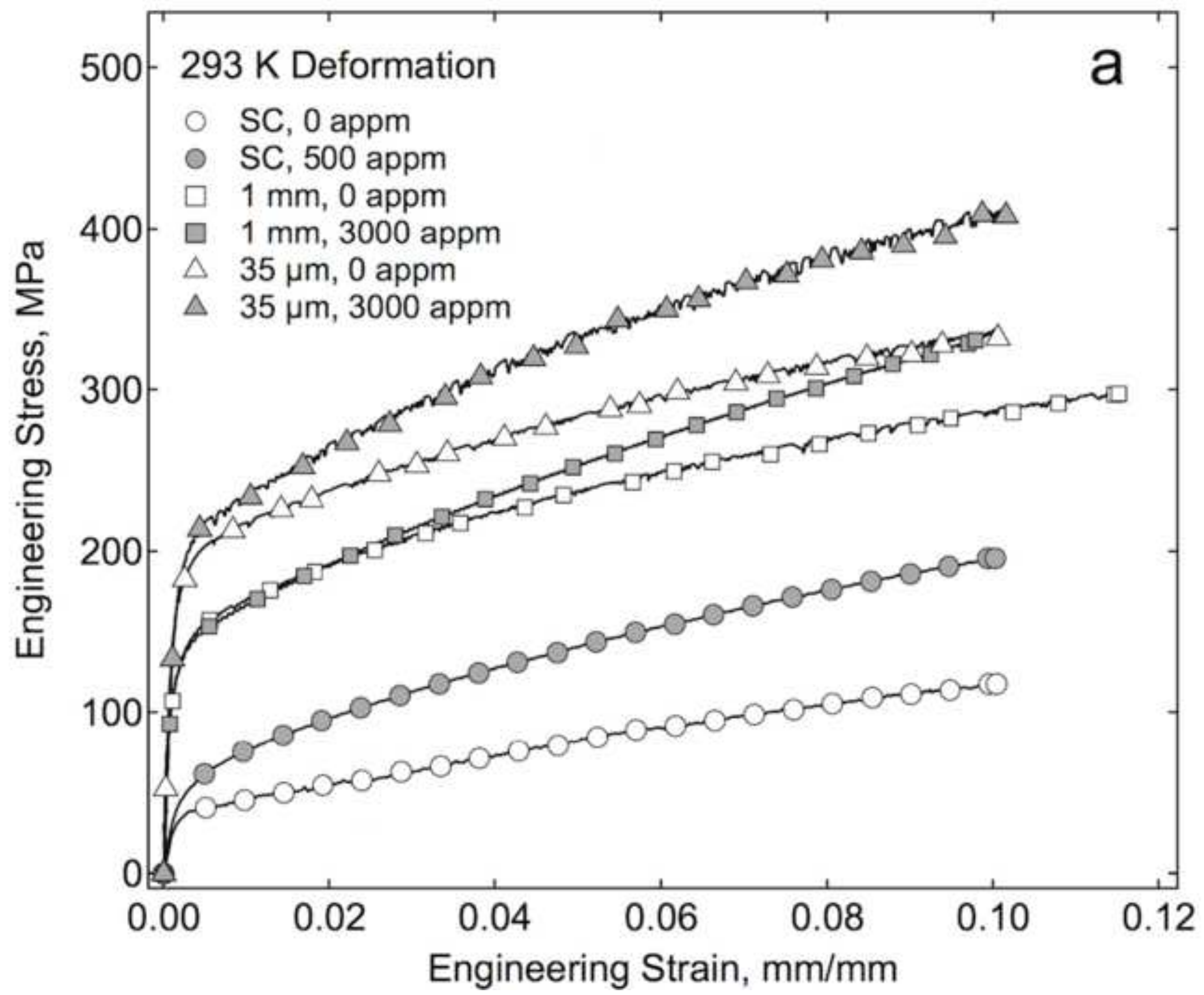




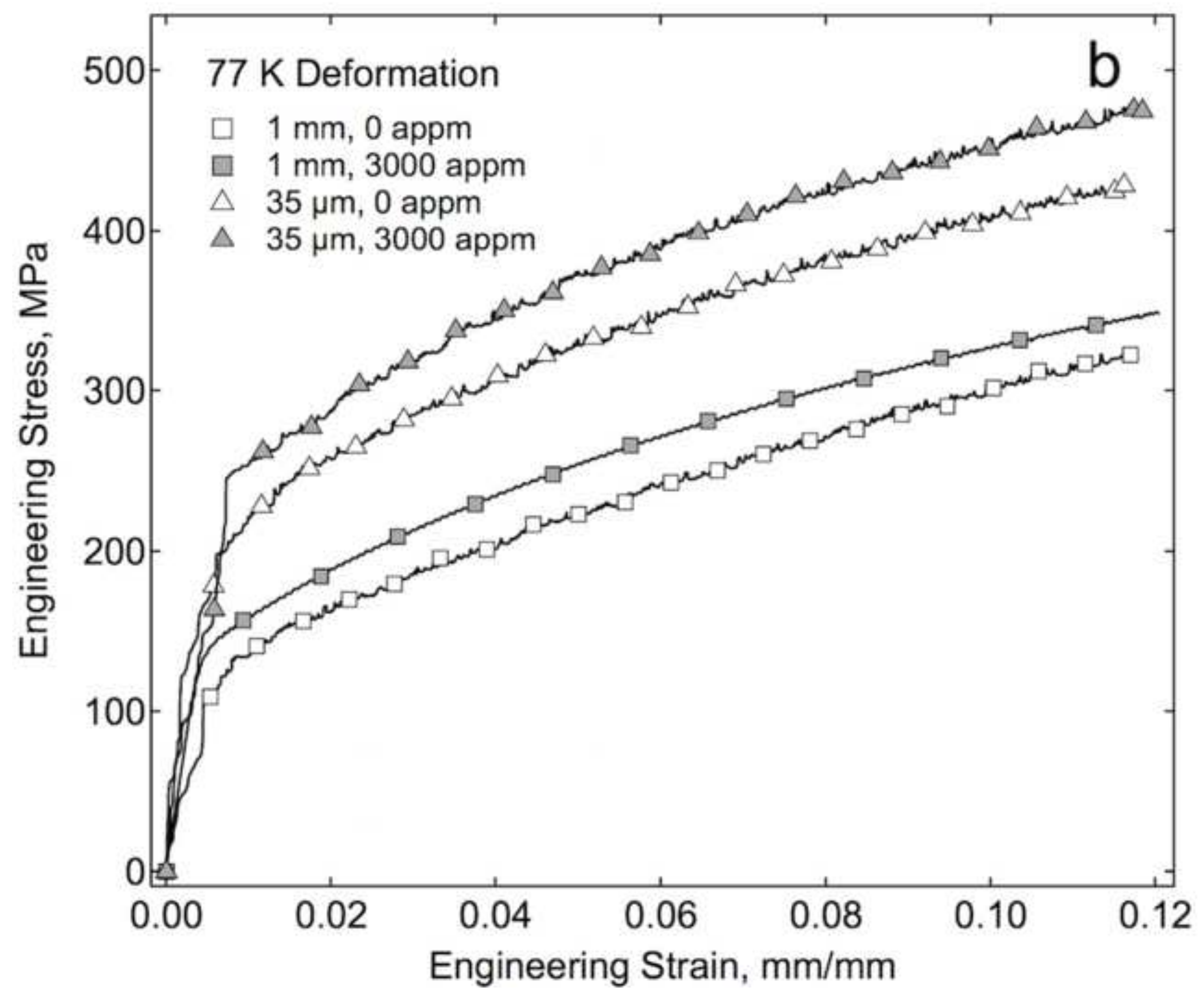




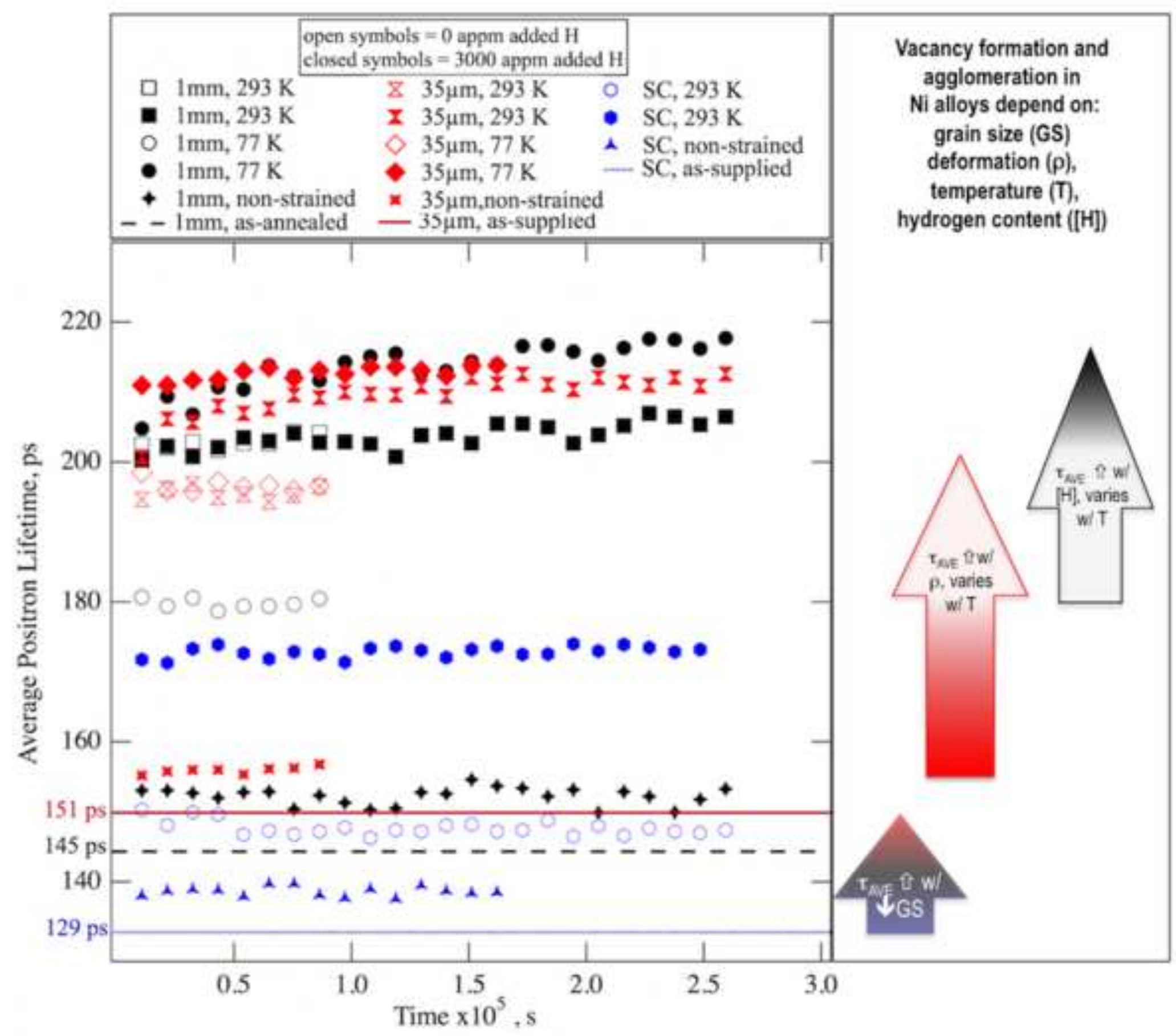

